\title{
Pulmonary Tuberculosis with Longitudinally Extensive Transverse Myelitis
}

\author{
Kiran Kumar Ramineni ${ }^{1}$ Ravi Kanth Jakkani \\ ${ }^{1}$ Department of Neurology, Yashoda Super Speciality Hospital, \\ Malakpet, Hyderabad, Telangana, India \\ 2Department of Radiology, Yashoda Super Speciality Hospital, \\ Malakpet, Hyderabad, Telangana, India \\ ${ }^{3}$ Department of Pulmonology, Yashoda Super Speciality Hospital, \\ Malakpet, Hyderabad, Telangana, India \\ J Neurosci Rural Pract 2020;11:178-182
}

\author{
B. V. G. Swamy ${ }^{3}$ Sravan Kumar M. ${ }^{2}$
}

\author{
Abstract \\ Keywords \\ - corticosteroids \\ - longitudinally \\ extensive transverse \\ myelitis \\ - pulmonary \\ tuberculosis
}

\begin{abstract}
Address for correspondence Ravi Kanth Jakkani, MD, FRCR, Department of Radiology, Yashoda Super Speciality Hospital, Near Nalgonda Cross Roads, Malakpet, Hyderabad, Telangana 500036, India (e-mail: ravikanthjakkani@gmail.com).
\end{abstract}

\section{Introduction}

Longitudinally extensive transverse myelitis is characterized by contiguous inflammatory lesions of spinal cord extending to three or more vertebral segments. Various etiologies include neuromyelitis optica spectrum disorders (NMOSD), infections, acute disseminated encephalomyelitis, connective tissue disorders, etc. ${ }^{1}$ Mycobacterium tuberculosis infection is a rare cause of transverse myelitis. Existing literature suggests a potential link between M. tuberculosis infection and NMOSDs. ${ }^{2,3}$ We report a case of aquaporin 4 antibody negative LETM without optic neuritis in a young man with active pulmonary tuberculosis.

\section{Case Presentation}

A 16-year-old boy admitted to our hospital with a history of weakness and numbness of both lower limbs of 3 weeks duration. He had history of urinary retention followed by urgency. No history of headache, seizures, or altered sensorium. No history of visual symptoms or upper limb involvement. No recent vaccination or travel. He had history of low-grade fever, dry coughs for the past 15 days. No history of weight loss or hemoptysis. No history of vomiting, hiccups, rash, pruritus, and joint pains. No family history of significant neurological illness. Birth and developmental history was normal and uneventful.

On neurological examination, he had normal higher intellectual functions. Fundus examination was normal. No evidence of any cranial nerve involvement. Speech was normal. Power was grade 5/5 in upper limbs and grade $3 / 5$ in both lower limbs. Brisk deep tendon reflexes in lower limbs with bilateral extensor plantar. Sensory examination revealed sensory level over the chest at $\mathrm{T} 4$ with impaired sensation below. There was no neck stiffness, spinal deformity, or tenderness. At the time of admission, his vitals were within normal limits. His height and weight were $162 \mathrm{~cm}$ and $60 \mathrm{~kg}$, respectively. General and other system examination were unremarkable except for bronchial breath sounds on right side.

Based on the history and clinical findings, the provisional diagnosis of noncompressive myelopathy with respiratory tract infection was made, and he was evaluated further.

Magnetic resonance imaging (MRI) of the spine plain and contrast revealed long-segment T2 and short T1 inversion recovery cord hyperintensity extending from C7 to D7 level with mild cord swelling (-Fig. 1). Postcontrast study did not reveal abnormal enhancement ( - Fig. 2). Screening of MRI of the brain was unremarkable. MRI findings were suggestive of long-segment extensive transverse myelitis. In view of the respiratory symptoms prior to starting 

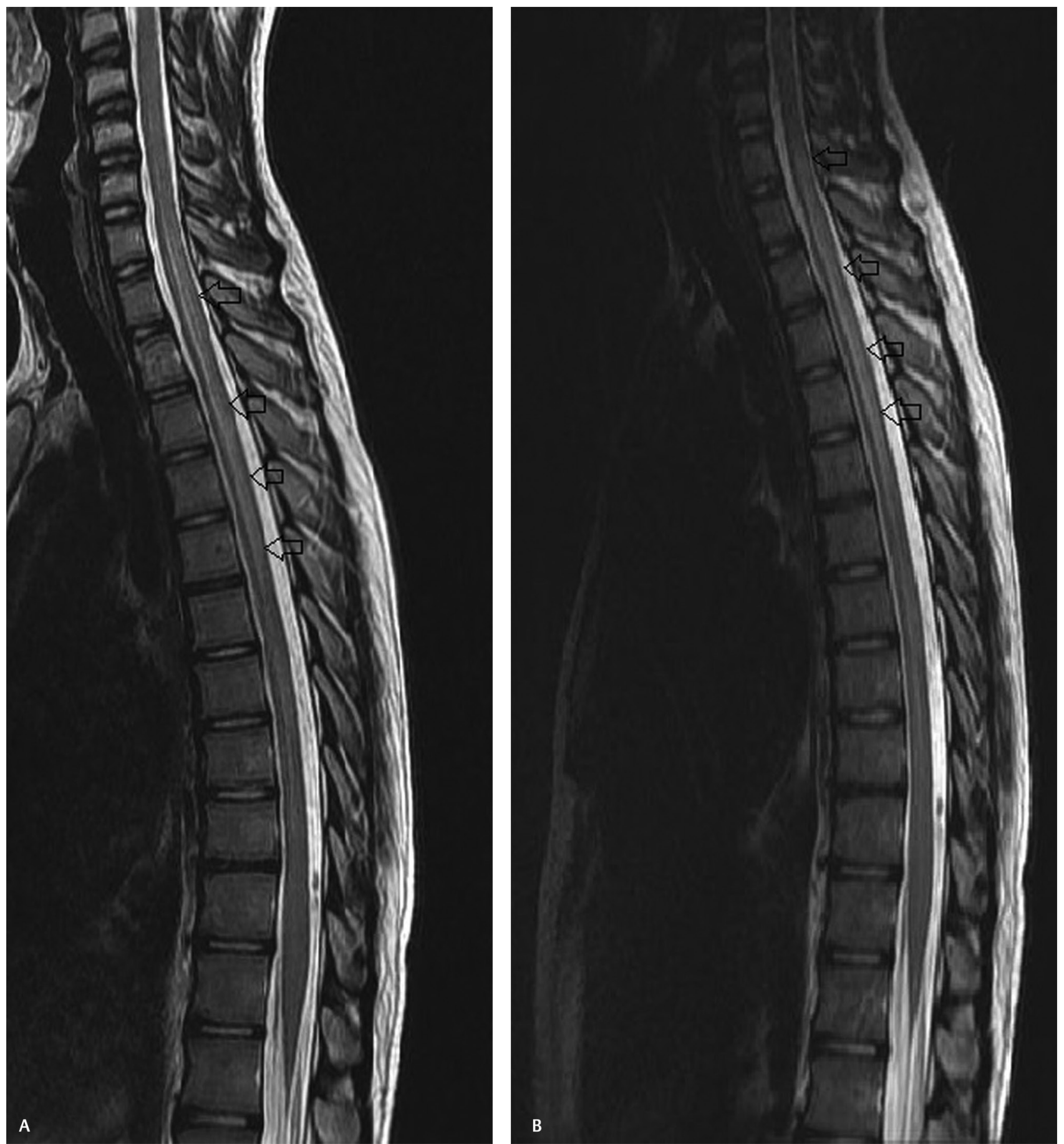

Fig. 1 (A, B) Sagittal T2-weighted images of the spine showing long-segment cord hyperintensity extending from C7 to D7 level.

intravenous steroids, he was further evaluated with chest radiograph which showed patchy infiltrates in right upper and mid-zones. Computed tomography (CT) of the chest revealed multiple clustered centrilobular nodules and tree in bud opacities with thin cavity in right upper lobe ( - Fig. $\mathbf{3}$ ). Bronchoalveolar lavage (BAL) was done and showed mixed inflammatory cells mostly histiocytes, few neutrophils, and lymphocytes admixed with columnar cells and occasional squamous cells in mucoid background. Acid fast bacilli were seen and gene Xpert MTB/RIF (mycobacterium/rifampicin) revealed $M$. tuberculosis and resistance to rifampicin was not found confirming the diagnosis of pulmonary tuberculosis. We refrained from culture investigation as it would have taken much longer time. Hemogram, serum creatine, electrolytes, serum calcium, serum B12 levels, and liver function tests were within normal limits. Erythrocyte sedimentation rate was elevated $40 \mathrm{~mm} / 1$ hour. Human immunodeficiency virus (HIV)-I and HIV-II were nonreactive. Serum angiotensin converting enzyme levels were within normal limits. Antinuclear antibody profile, rheumatoid arthritis factor, cytoplasmic antinuclear cytoplasmic antibodies, and perinuclear antinuclear cytoplasmic antibodies were negative. Serum 

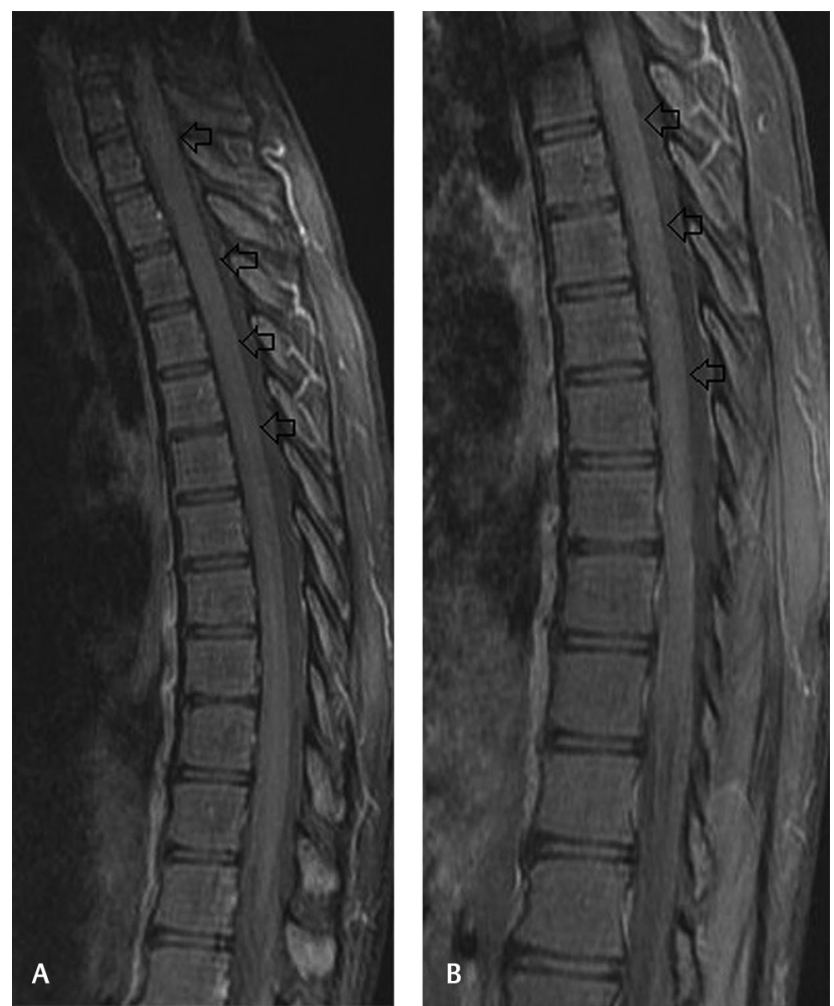

Fig. 2 (A, B) Sagittal postcontrast fat-saturated T1-weighted images showing no abnormal cord enhancement.
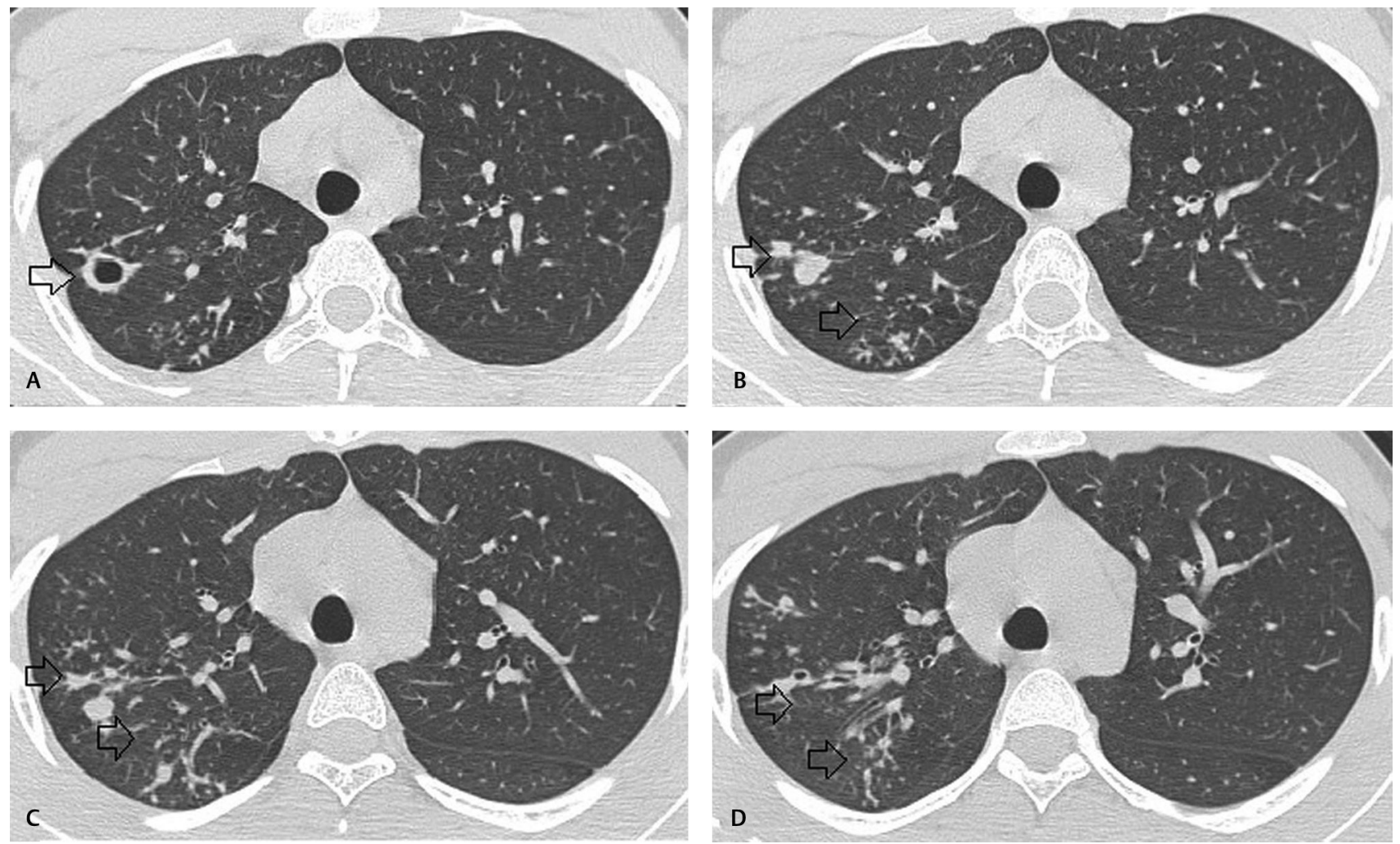

Fig. 3 (A-D) Axial computed tomography sections of lung showing multiple clustered centrilobular nodules and tree in bud opacities with thin cavity in right upper lobe.

aquaporin 4 antibody (NMO IgG) was negative. Electrocardiogram, two-dimensional echocardiogram, and ultrasound of the abdomen were unremarkable. Bilateral visual evoked potentials were normal.

Cerebrospinal fluid (CSF) analysis revealed mild elevation of protein $52 \mathrm{mg} / \mathrm{dL}$ with normal glucose level of $65 \mathrm{mg} / \mathrm{dL}$, cell count of 5 cells/cumm, no atypical cells, adenosine deaminase level of $4 \mathrm{U} / \mathrm{L}$, and polymerase chain reaction (PCR) for tuberculosis was negative. Aquaporin 4 antibody was negative. Herpes simplex viruses I and II PCR were also negative.

Final diagnosis of long-segment extensive transverse myelitis with pulmonary tuberculosis was made. He was treated with intravenous Solu-Medrol $1 \mathrm{~g} / \mathrm{d}$ for 3 days followed by oral prednisolone $1 \mathrm{mg} / \mathrm{kg} / \mathrm{d}$ tapered over the next 6 weeks. Four-drug antitubercular therapy (ATT) regimen (isoniazid, rifampicin, pyrazinamide, and ethambutol) was added simultaneously.

At 1 month follow-up, neurological deficits improved significantly except for mild bladder symptoms (urgency). Fever, cough subsided, and no adverse drug reactions observed. MRI of the spine at 3 months follow-up revealed complete resolution of cord edema ( - Fig. 4 ).

\section{Discussion}

Pulmonary and extrapulmonary tuberculoses are common in endemic countries like India. Clinical manifestations are highly variable and at times quite nonspecific. CNS

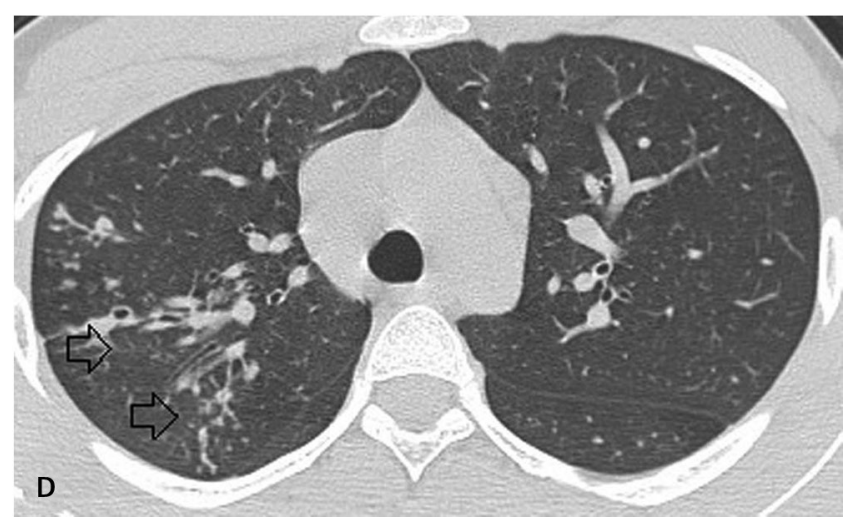



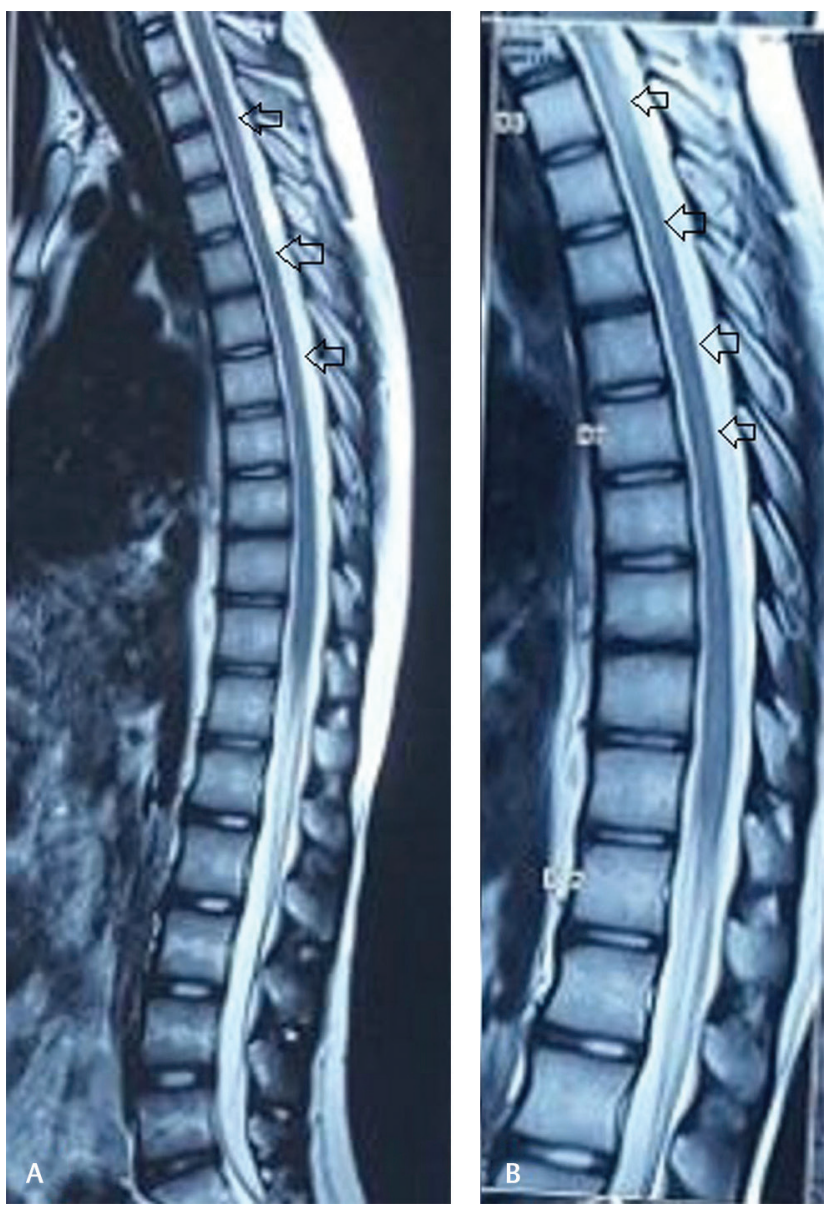

Fig. 4 (A, B) Posttreatment follow-up magnetic resonance imaging with sagittal T2-weighted images showing significant resolution of cord hyperintensity.

manifestations of tuberculosis include meningitis, tuberculomas, abscess, pachymeningitis, spinal arachnoiditis, and myelitis. Tuberculous myelitis is relatively uncommon and can be infective or immune medicated. ${ }^{4}$

Cervicodorsal regions are the most common site involved in tuberculous myelitis. Although rare in children, holocord involvement was also described in occult disseminated tuberculosis. ${ }^{5}$ As LETM is the cardinal manifestation of NMOSDs, thorough evaluation should be done for early diagnosis and for the possible secondary etiologies including infections, connective tissue disorders, etc. Serum aquaporin 4 antibody positivity is highly variable and can be negative in the context of classical clinical features. ${ }^{6}$ MRI of the spine and brain with contrast helps in the diagnosis and planning further workup. Our patient had classical MRI findings of LETM. Serum and CSF aquaporin 4 antibody workup was negative. In view of the respiratory symptoms prior to starting intravenous steroids, he was further evaluated with chest radiograph, CT of the chest followed by BAL, which clinched the diagnosis of active pulmonary Koch's.

Significant association between neuromyelitis optica and pulmonary tuberculosis was reported in a case-control study by Zatjirua et al. ${ }^{7}$ Genetic susceptibility of the host, strain variation of the pathogen, and endemicity of tuberculosis might determine the strength of the association.
As CSF analysis in our patient did not reveal features, suggestive of active infection likely mechanism of myelitis is immunological. Zhang et al reported similar phenomenon in a young female. ${ }^{8}$ Wisniewski Bloom reported demyelination and inflammatory response after injecting BCG into sensitized guinea pigs. ${ }^{9}$ Mycobacterium tuberculosis may have common antigens with myelin basic protein. Hughes and Mair reported acute necrotic myelopathy with pulmonary tuberculosis and hypothesized delayed hypersensitivity reaction caused by MTB might be the cause of demyelination. ${ }^{10}$

Our patient did not meet the criteria for neuromyelitis optica during initial admission and throughout follow-up. However, in view of active pulmonary tuberculosis, he was treated with combination of ATT with steroids. He had good improvement and no relapses during 1-year follow-up. As the myelitis clearly preceded the initiation of ATT, it is unlikely due to paradoxical therapeutic response. Feng et al reported better outcome and reduced relapse rates in neuromyelitis optica patients with the addition of antituberculosis treatment to the conventional regimens. ${ }^{11}$

\section{Conclusion}

LETM is a potentially treatable neurological disorder with significant morbidity. Parenteral and oral corticosteroids are the mainstay of treatment protocols. Thorough evaluation of the co-occurring pulmonary and extrapulmonary tuberculoses should be done; more so in tuberculosis endemic areas like India. Timely initiation of combination of steroids with antituberculosis medication may result in better long-term outcome in selected patients.

\section{Funding \\ None.}

\section{Conflict of Interest}

None declared.

\section{References}

1 Jain RS, Kumar S, Mathur T, Tejwani S. Longitudinally extensive transverse myelitis: a retrospective analysis of sixty-four patients at tertiary care center of North-West India. Clin Neurol Neurosurg 2016;148:5-12

2 Silber MH, Willcox PA, Bowen RM, Unger A. Neuromyelitis optica (Devic's syndrome) and pulmonary tuberculosis. Neurology 1990;40(6):934-938

3 Rafai MA, Boulaajaj FZ, Gynerane M, El Moutawakkil B, Slassi I. Devic-like syndrome in the course of pulmonary tuberculosis. Acta Neurol Belg 2010;110(2):196-200

4 Sahu SK, Giri S, Gupta N. Longitudinal extensive transverse myelitis due to tuberculosis: a report of four cases. J Postgrad Med 2014;60(4):409-412

5 Alkan G, Emiroğlu M, Kartal A, Peru H, Koplay M. Occult disseminated tuberculosis with holocord longitudinally extensive transverse myelitis: a rare phenomenon in a child. J Pediatr Neurosci 2017;12(3):259-261

6 Tenembaum S, Chitnis T, Nakashima I, et al. Neuromyelitis optica spectrum disorders in children and adolescents. Neurology 2016;87(9, suppl 2) :S59-S66 
7 Zatjirua V, Butler J, Carr J, Henning F. Neuromyelitis optica and pulmonary tuberculosis: a case-control study. Int J Tuberc Lung Dis 2011;15(12):1675-1680

8 Zhang Y, Zhu M, Wang L, Shi M, Deng H. Longitudinally extensive transverse myelitis with pulmonary tuberculosis: two case reports. Medicine (Baltimore) 2018;97(3):e9676

9 Wisniewski HM, Bloom BR. Primary demyelination as a nonspecific consequence of a cell-mediated immune reaction. J Exp Med 1975;141(2):346-359
10 Hughes RA, Mair WG. Acute necrotic myelopathy with pulmonary tuberculosis. Brain 1977;100(2):223-238

11 Feng YQ, Guo N, Huang F, Chen X, Sun QS, Liu JX. Antituberculosis treatment for Devic's neuromyelitis optica. J Clin Neurosci 2010;17(11):1372-1377 